\title{
The new ACCF/AHA guidelines on the treatment of dyslipidemia: pros
}

\author{
Raffaele De Caterina
}

Received: 5 September 2014 / Accepted: 14 November 2014/Published online: 13 December 2014 (C) SIMI 2014

\section{Introduction: the novelty introduced by the latest 2013 ACC/AHA guideline on the treatment of blood cholesterol}

The new 2013 ACC/AHA Guidelines in hypercholesterolemic adults [1] have spurred major debates in the medical community as well as in the lay press. Compared with the previous report of the National Cholesterol Education Program (NCEP) Expert Panel on Detection, Evaluation, and Treatment of High Blood Cholesterol in Adults (Adult Treatment Panel III) [2] and with the 2011 ESC Guidelines in the same area [3], these guidelines have identified four major groups of patients for whom cholesterol-lowering HMG-CoA reductase inhibitors, or statins, have the greatest chance of preventing stroke and heart attacks, and suggest treating them from the very beginning with a moderate- high-intensity dose. The guidelines also largely emphasize the importance of adopting a heart-healthy lifestyle to prevent and control high blood cholesterol.

These guidelines represent a departure from previous documents because they do not focus on specific target levels of low-density lipoprotein cholesterol, commonly known as low-density lipoprotein (LDL), or "bad" cholesterol, although the definition of optimal LDL cholesterol has not changed. Instead, they focus on defining groups for whom LDL lowering is proven to be most beneficial. The

R. De Caterina $(\square)$

Institute of Cardiology, "G. d'Annunzio" University, Chieti-

Pescara, C/o Ospedale SS, Annunziata, Via dei Vestini,

66013 Chieti, Italy

e-mail: rdecater@unich.it

R. De Caterina

G. Monasterio Foundation, Pisa, Italy guidelines focus only on the use of statins, after a detailed review of other cholesterol-lowering drugs. Statins were chosen because their use has resulted in the greatest benefit and the lowest rates of safety issues. No other cholesterollowering drug is as effective as statins, although there is a role for other cholesterol-lowering drugs, for example, in patients who suffer side effects from statins.

The guidelines emphasize that, although other strategies for using drug therapy to reduce atherosclerotic cardiovascular disease (ASCVD) events have been advocated, including treat-to-cholesterol target, lowest is best, and risk-based treatment approaches, only one approach has been de facto evaluated in multiple randomized clinical trials (RCTs) - the use of fixed doses of cholesterol-lowering drugs to reduce ASCVD risk. Because the overwhelming body of evidence comes from statin RCTs, the Expert Panel appropriately focused on these statin RCTs to develop evidence-based guidelines for the reduction of ASCVD risk.

\section{Recommending a practical approach to high LDL-c patients}

The new ACC/AHA guidelines [1] recommend moderateor high-intensity statin therapy for these four groups:

- Patients who have cardiovascular disease;

- Patients with an LDL level of $190 \mathrm{mg} / \mathrm{dL}$ or higher;

- Patients with Type 2 diabetes who are between 40 and 75 years of age; and

- Patients with an estimated 10 year risk of cardiovascular disease of 7.5 percent or higher who are between 40 and 75 years of age (the report provides formulas for calculating the 10 year risk). 
In addition to identifying patients most likely to benefit from statins, the guidelines outline the recommended intensity of statin therapy for different patient groups. Rather than use a "lowest is best" approach that combines a low dose of a statin drug along with several other cholesterol-lowering drugs, the panel found that it can be preferable to focus instead on a healthy lifestyle along with a higher dose of statins, eliminating the need for additional medications. While the focus for years has been on getting the LDL low, these guidelines now say that how you get the LDL low is important.

\section{Why staying out of the target concept?}

The concept of treating to a target level of LDL cholesterol comes from the commonly used and sensible practice of calibrating the intensity of any preventive treatment to the level of the baseline risk, with the awareness that any pharmacological treatment has downsides, and therefore, going down across the risk spectrum, one should-sooner or later-find an "equipoise", a level of risk below which the expected benefits of a treatment are outweighed by its inherent risk. This risk assessment-based approach is used commonly in most areas of Medicine, for example in deciding whether or not to prescribe aspirin in people without evidence of previous cardiovascular disease [4]. Setting more and more stringent criteria when the risk of future cardiovascular events gets higher also helps remind doctors that their alertness should be maximum in subjects at highest risk, and an intensified therapy has to be pursued in higher risk categories.According to these principles, the 2011 ESC guidelines [3], in a specific paragraph named "Treatment targets", recommended a tailoring of treatment according to the baseline risk, defined according to the SCORE calculations [5], assessing the 10-year risk of fatal cardiovascular disease. According to this, in patients at very high $\mathrm{CV}$ risk (a SCORE level $>10 \%$ ), a target of $<70 \mathrm{mg} / \mathrm{dL} \quad(1.8 \mathrm{mmol} / \mathrm{L})$ is recommended; but more lenient targets are recommended for patients at high $\mathrm{CV}$ risk (SCORE level $>5 \%$ ) - in this case $<100 \mathrm{mg} / \mathrm{dL}$ or $2.5 \mathrm{mmol} / \mathrm{L})$ - , and even more lenient targets for patients at moderate risk (SCORE level 1.5) - in this case $115 \mathrm{mg} /$ $\mathrm{dL}$, or $3.0 \mathrm{mmol} / \mathrm{L}$.

The explicit justification for this approach is, however, vague: "The overall guidelines on CVD prevention in clinical practice strongly recommend modulating the intensity of the preventive intervention according to the level of the total $\mathrm{CV}$ risk. Therefore, the targets should be less demanding when the total CV risk decreases from very high to high or moderate" [3]. Indeed those very same ESC guidelines cite that the most recent Cholesterol Treatment Trialists' Collaboration (CTT) meta-analysis of several trials involving $>170,000$ patients, which confirmed the dose-dependent reduction in CVD with LDL-C lowering [6]. The Interpretation paragraph in the abstract of that publication explicitly states: "Further reductions in LDL cholesterol safely produce definite further reductions in the incidence of heart attack, of revascularisation, and of ischaemic stroke, with each $1.0 \mathrm{mmol} / \mathrm{L}$ reduction reducing the annual rate of these major vascular events by just over a fifth. There was no evidence of any threshold within the cholesterol range studied, suggesting that reduction of LDL cholesterol by $2-3 \mathrm{mmol} / \mathrm{L}$ would reduce risk by about 40-50\%" [6].

Although side effects of statins - by far the best studied approach to reduce LDL cholesterol-are known to be dose-dependent [7], analysis of risk associated with statin treatment also in low-risk populations (with 5-year risk of major vascular events lower than $10 \%$ ) has shown a persistence of benefits outweighing the risk [8]. Although benefit may not include a reduction in mortality in primary prevention [9], there still appears to be an effect on important non-fatal events such as non-fatal myocardial infarction, stroke, or coronary revascularization [8]. In addition to being associated with a favorable efficacysafety balance, with the emergence of generic high-potency statins, such as atorvastatin and-soon-rosuvastatin, statin therapy is also increasingly becoming cost-effective, and most of thecounterpoints for a wide adoption of their use appear to be minimized [10].

It is true that safety, as reported from clinical trials, is underestimated because of the upstream exclusion from trials of patients experiencing side effects [11]. But statin safety can in most cases easily be assessed in a run-in period [7], so that the justification for withholding a highpotency weapon, such as atorvastatin $40-80 \mathrm{mg} /$ day or rosuvastatin $20-40 \mathrm{mg} / \mathrm{day}$, in healthy persons at risk because they have already reached their lenient target of $115 \mathrm{mg} / \mathrm{dL}$ (3 mmol/L) of LDL cholesterol [3], appears not to be scientifically sound. Incidentally, the reduction of such target value from the previous one of $130 \mathrm{mg} / \mathrm{dL}$ [2] appears more a kind of compromise to accommodate new facts into an old scheme, than an act of courage to abandon old paradigms. Given the absence of data on titration of drug therapy to specific goals, no recommendations are, therefore, made in the latest ACC/AHA Guidelines [1] for or against specific LDL cholesterol or non-HDL cholesterol goals for the primary or secondary prevention of ASCVD.

\section{The impact of this change in paradigm}

The likely impact of the new ACC/AHA recommendations is that-if doctors will adhere to these recommendations- 
more people who would benefit from statins are going to be on them. Doctors may also consider switching some patients to a higher dose of statins to derive greater benefit as a result of this approach focusing on the immediate use of a moderate- or high-intensity statin treatment in most individuals at risk. I support this position.

Conflict of interest None.

\section{References}

1. Stone NJ, Robinson JG, Lichtenstein AH et al (2014) 2013 ACC/ AHA guideline on the treatment of blood cholesterol to reduce atherosclerotic cardiovascular risk in adults: a report of the American college of cardiology/American HEART Association Task Force on Practice Guidelines. Circulation 129:S1-S45

2. Third Report of the National Cholesterol Education Program (NCEP) (2002) Expert panel on detection, evaluation, and treatment of high blood cholesterol in adults (Adult Treatment Panel III) final report. Circulation 106:3143-3421

3. Reiner Z, Catapano AL, De Backer G et al (2011) ESC/EAS Guidelines for the management of dyslipidaemias: the Task Force for the management of dyslipidaemias of the European Society of Cardiology (ESC) and the European Atherosclerosis Society (EAS). Eur Heart J 32:1769-1818
4. Halvorsen S, Andreotti F, Ten Berg JM et al (2014) Aspirin therapy in primary cardiovascular disease prevention: a position paper of the european society of cardiology working group on thrombosis. J Am Coll Cardiol 64:319-327

5. Conroy RM, Pyorala K, Fitzgerald AP et al (2003) Estimation of ten-year risk of fatal cardiovascular disease in Europe: the SCORE project. Eur Heart J 24:987-1003

6. Baigent C, Blackwell L, Emberson J et al (2010) Efficacy and safety of more intensive lowering of LDL cholesterol: a metaanalysis of data from 170,000 participants in 26 randomised trials. Lancet 376:1670-1681

7. Desai CS, Martin SS, Blumenthal RS (2014) Non-cardiovascular effects associated with statins. Bmj 349:g3743

8. Mihaylova B, Emberson J, Blackwell L et al (2012) The effects of lowering LDL cholesterol with statin therapy in people at low risk of vascular disease: meta-analysis of individual data from 27 randomised trials. Lancet 380:581-590

9. Ray KK, Seshasai SR, Erqou S et al (2010) Statins and all-cause mortality in high-risk primary prevention: a meta-analysis of 11 randomized controlled trials involving 65,229 participants. Arch Intern Med 170:1024-1031

10. Blaha MJ, Nasir K, Blumenthal RS (2012) Statin therapy for healthy men identified as "increased risk". JAMA 307:1489-1490

11. Redberg RF, Katz MH (2012) Healthy men should not take statins. JAMA 307:1491-1492 